\title{
Konstruktionen medizinischer Wirklichkeit
}

\section{Sebastian Till Hartwig}

Nicht nur zu Zeiten aktueller - und teils absurder - Debatten um

Immunisierungsstrategien sowie intensivierter Gesundheitsdiskurse begleitet uns ein oft unbemerktes ,Gespenst'. Und zwar eines, das zu einem durchaus spannenden Gegenstand (wissens-)soziologischer Analysen werden kann: das Vitamin C. Denn für das allgemein vorherrschende Alltagswissen über Vitamin C - in Kurzform: eine möglichst hohe Ein- bzw. Aufnahme kann das Immunsystem stärken und so etwa Erkältungen vorbeugen - gibt es keine solide wissenschaftliche Evidenz. Viel eher ist der ,Mythos‘ um das Vitamin Ergebnis einer strategisch geplanten Fabrikation von Wissen.

\section{Nachweisbarkeit}

Die medizinische Wirkung von Vitamin C-Präparaten auf Erkältungen kann bis heute wissenschaftlich nicht eindeutig belegt werden. Ganz im Gegenteil: In den letzten Jahren wurden mehr und mehr Studien publiziert, die deren vermeintliche Wunderwirkung deutlich relativieren. Oder sogar belegen, dass Häufigkeit, Dauer und Schwere von Erkältungen nicht oder nur sehr geringfügig durch die regelmäßige Einnahme von Vitamin $\mathrm{C}$ beeinflusst werden können. In der gegenwärtigen medizinischen Fachdiskussion ist man sich weitgehend einig: Zweifellos spielt Vitamin C eine lebensnotwendige Rolle für den menschlichen Stoffwechsel und das Immunsystem; jedoch deckt sich der ,normale“ Bedarf - ohne etwa Hochleistungssport zu betreiben - vollständig durch eine ausgewogene Ernährung (auch wenn hier sicherlich auslegungsbedürftig ist, welche Ernährung nun als ,ausgewogen' gilt). Und dennoch: Das Geschäft mit dem Vitamin floriert; nach Magnesium ist es das zweitbeliebteste Verkaufsprodukt unter den Nahrungsergänzungsmitteln und Vitaminpräparaten.

\section{Woher also der weitverbreitete (Irr-)Glaube an die Extraportion Vitamin C?}

Schon ein kurzer Blick auf nur einige Etappen seiner Erfolgsgeschichte kann ein wenig Licht ins Dunkel bringen: Bereits im 18. Jahrhundert wurde nach Behandlungsmethoden gegen die Seefahrer- und Expeditionskrankheit Skorbutgesucht. In den 1740er Jahren konnte (durch den schottischen Schiffsarzt James Lind) nachgewiesen werden, dass sich der Verzehr von Zitrusfrüchten positiv auf die Behandlung von Skorbut auswirkt. Dass es sich dabei um eine Vitaminmangelkrankheit handelt, sollte aber noch bis in das 20. Jahrhundert hinein unbekannt bleiben. 
In den 1920er Jahren gelang es Biochemikern erstmals, aus Pflanzenextrakten und Zitronensaft die sogenannte „Hexuronsäure“ zu gewinnen - heute besser bekannt als „Ascorbinsäure“ oder „Vitamin C“. Für diese Entdeckungen erhielt Albert SzentGyörgyi im Jahr 1937 den „Nobelpreis für Physiologie oder Medizin“ (der Biochemiker Charles Glen King, der parallel dazu die gleichen Entdeckungen für sich beanspruchte, erhielt keine vergleichbare Auszeichnung). Nur wenige Jahre später, in den frühen 1930ern, entdeckte der Schweizer Chemiker Tadeus Reichstein eine Methode, Vitamin C zu synthetisieren: der Durchbruch zur industriellen Massenproduktion.

Im Jahr 1933 patentierte Reichstein seine Methode und wandte sich an das schweizerische Pharmaunternehmen Hoffmann-La Roche. Einblicke in das Historische Archiv Roche belegen, dass der damalige Forschungschef des Unternehmens, Markus Guggenheim, jedoch keinen Bedarf für die künstliche Herstellung sah: „Erwachsenen dürfte in der Norm genügend Vitamin C mit frischem Gemüse, Obst und dergleichen zukommen“. Um die neue

Synthetisierungsmöglichkeit in ökonomischen Profit umzuwandeln, galt es also, den Bedarf erst noch zu schaffen.

\section{Die Vermarktung}

Wie aber einen Bedarf schaffen, wo keiner besteht; einen Absatzmarkt, wo kein Umsatz in Aussicht ist? Als viel zu selten auftretendes Krankheitsbild würde Skorbut allein nicht ausreichen. Die Lösung findet sich in einer überaus wirkungsvollen Marketingstrategie: in der Erdichtung eines neuen Krankheitsbildes. Unter der Bezeichnung „C-Hypovitaminose“ wurde Patient*innen eine vermeintliche Unterversorgung an Vitamin C diagnostiziert. Das Heilmittel: die von La Roche unter dem Markennamen Redoxon angebotene, reine Ascorbinsäure. Gewiss ist dies kein Einzelfall der erfolgreichen Erfindung neuer Krankheitsbilder in der Geschichte der Pharmazeutik; besonders sind aber die Einblicke in die interne Korrespondenz des Unternehmens.

Aus der sogenannten „Propaganda-Abteilung “von La Roche (heute würde wohl politisch neutraler und moderner von „Marketing-Abteilung“ gesprochen) wird 1935 in einem Brief an die Firmendirektion in Basel zu dem „Gespenst der allgemein drohenden C-Hypovitaminose“gefordert: „Redoxon muss also jedenfalls als schlagkräftige Firmenpropaganda betrachtet und entsprechend ausgeschlachtet werden." Auch kritisch positionierte und wissenschaftlich arbeitende Ärzte sollten überzeugt und so „das Gros der praktischen Ärzte aus Vitaminungläubigen zu Vitamingläubigen bzw. Vitaminschreibern" gemacht werden.

Nachdem diese in martialische Metaphern und Missionierungsrhetorik gehüllte Marketingstrategie erste Erfolge zeigte, wurden die Präparate nicht mehr nur kranken und Risiko-Patienten verschrieben, sondern auch an als „vollkommen gesund“ geltende Menschen verkauft. Denn ein profitabler Absatz konnte nur erreicht werden, 
wenn sich eine regelmäßige Einnahme von Redoxon auch auf lange Sicht durchsetzen würde - und dazu müsste eben, wie die „Propaganda-Abteilung“ betonte, „etwas Hokuspokus gemacht“" werden.

\section{Die Methode}

Um diesen Plan zu realisieren, (er-)fand das Unternehmen eine eigens zur Kontrolle des Vitamin C-Stoffwechsels dienende Methode: Durch eine im Urin der Patient*innen aufgelöste Tablette (Dichlorphenolindophenol <Roche ) konnte anhand der Färbung ein vermeintlicher Vitamin C-Mangel diagnostiziert werden. Mit der Einrichtung dieser Diagnosemethode wurde nicht nur der Verkauf von Redoxon weiter angetrieben, sondern auch der Glaube an die wissenschaftliche Belegbarkeit seiner Wirkung gefestigt. Unter leicht verständlicher Anleitung sollte „selbst der einfachste Mann aus dem Volke“in der Lage sein, einen vermeintlichen Zusatzbedarf an Vitamin C zu erkennen. Der Vorschlag:

„Ein spannender Prospekt über die Schädlichkeit der C-Hypovitaminosen und wie wir alle davon bedroht sind! [...] Entgegen der Vorschrift, versucht man es gleich mit dem Harn [...]. Entfärbt nicht! Böses Omen! Jetzt aber los mit Redoxon!“

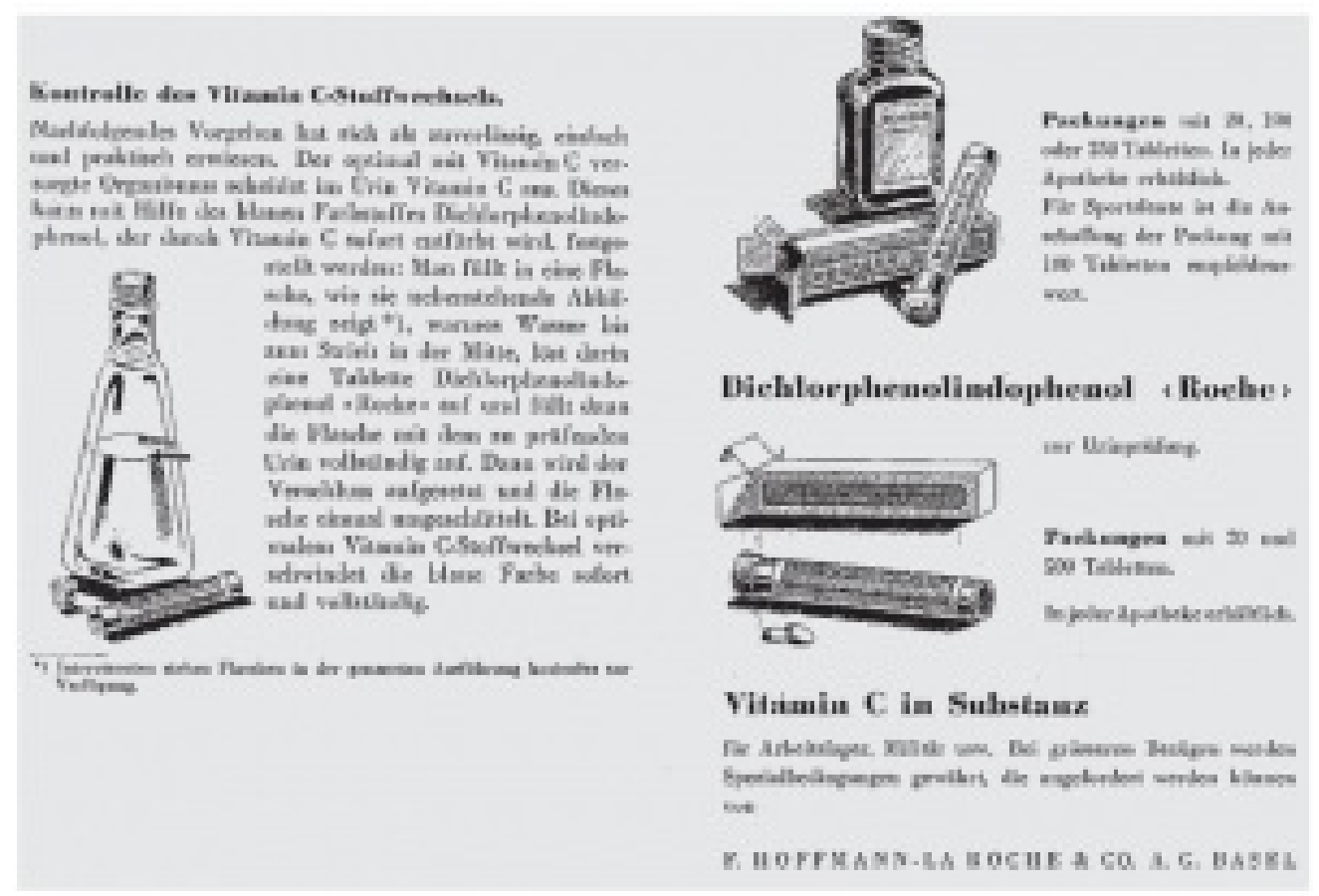

Anleitung zur Therapierung der erdichteten „C-Hypovitaminose“ (Abb. nach Bächi 2009: 141)

Auch die Einflussmöglichkeiten massenmedialer Berichterstattung wurden von der „Propaganda-Abteilung“ des Unternehmens genutzt: Die Schweizerische Medizinische Wochenschrift wurde ab 1933 mit jährlichen Druckkostenzuschüssen von La Roche zu einem Unterstützer ihrer Marketinginteressen gemacht. Die Höhe der Zuschüsse sollte von der Anzahl und Länge der für das Unternehmen nützlichen Publikationen abhängen - „Lobbyarbeit“ wäre heute vermutlich ein passender Begriff dafür. Begleitet wurde dieses Vorgehen von zahlreichen Werbe-, Vertriebs- und 
weiteren ,Propaganda'-Kampagnen. Vitamin C avancierte so zum allgemein anerkannten Modeprodukt und vermeintlichen Wundermittel. Und ist bis heute freilich nicht nur für die Firma La Roche - ein Millionengeschäft.

\section{Die Aufrechterhaltung}

Doch wie konnte sich der Glaube an die Extradosis Vitamin C so lange halten? Die gesellschaftlichen, wirtschaftlichen und politischen Bedingungen, unter denen die Erfolgsgeschichte des Redoxon in den 1930er Jahren ihren Lauf nehmen konnte, waren überaus komplex. Beat Bächi hat dazu in seiner 2009 erschienenen Dissertation bemerkenswerte historische Analysen vorgelegt. Das Interessante an einem Blick auf die Gegenwart ist jedoch, dass sich diese Erfolgsgeschichte noch immer fortschreibt. Der zahlreichen widerlegenden Studien und Publikationen unbeeindruckt scheint sich der einst als Vermarktungsstrategie verbreitete ,Mythos“ um die Wunderwirkung des Vitamin C weiter durchzusetzen.

Er ist nicht nur ein Residuum veralteten, strategisch platzierten und auch erdichteten („C-Hypovitaminose“) Wissens. Er wird vielmehr kontinuierlich aufrechterhalten durch massenmediale Bewerbung, Produktplatzierung und andere erfolgreiche Vermarktungsstrategien. Es wäre jedoch zu einseitig, nur auf die öffentlichkeitswirksamen und wirtschaftlich agierenden Akteure zu blicken. Denn schließlich wird die Maschinerie des Vitamin C-Mythos - das gilt ebenso für zahlreiche andere Nahrungsergänzungs- und Hausmittelchen - vor allem durch soziale Praktiken des Alltags angetrieben: durch das Kochen Vitamin C-reicher Speisen gleichermaßen wie durch die Einnahme von Präparaten bei Erkältungen bzw. zu deren Vorbeugung. Und natürlich durch den kommunikativen Austausch über diese Praktiken. So werden Wissen und Wirklichkeit in und durch gesellschaftliche Praxis (re-)konstruiert und auf Dauer gestellt. Eine Wirklichkeit und ein Wissen, die - auch trotz deren wissenschaftlicher Widerlegbarkeit - deshalb wirklich sind, weil sie es in ihren sozialen Konsequenzen sind. Ganz im Sinne des Thomas-Theorems: „If men define situations as real, they are real in their consequences“.

Als Erfolgsgeschichte pharmazeutischer Kommerzialisierung ist das Vitamin C gewiss kein Einzelfall. Wie Pharmaunternehmen durch Marketingkampagnen und Lobbyismus nicht nur zu enormem ökonomischem Profit gelangen (konnten), sondern auch zur Veränderung sozialer Wirklichkeit beitragen, zeigt sich in strukturell ähnlicher - wenn auch weitaus dramatischerer - Weise an der OpioidEpidemie in den USA. Die Nutzung höherer Absatzmöglichkeiten durch leichtfertige Diagnosen lässt sich vergleichbar an den Ritalin-Sammelklagen gegen den Pharmakonzern Novartis nachvollziehen.

Aus einer an der Entstehung und Tradierung von Wissen interessierten Perspektive bietet der Fall des Vitamin C eine Reihe - noch ausstehender - analytischer Anschlussmöglichkeiten. Neben der für die Wissenssoziologie so zentralen Frage, durch welche Prozesse „ein bestimmter Vorrat von » Wissen « gesellschaftlich etablierte »Wirklichkeit« werden konnte“, berührt er auch ein sehr asymmetrisches 
Verhältnis von wissenschaftlich begründetem Wissen und Alltagswissen. Hier zeigen sich aktualisierte wissenschaftliche Erkenntnisse (neuere Studien) weitestgehend wirkungslos gegen etablierte gesellschaftliche Überzeugungen; und es sind im Wesentlichen letztere, die soziale Praxis formen und somit - auch medizinische Wirklichkeit konstruieren. Von normativen Implikationen dieses Beispiels unberührt zeigt sich aber auch, dass die analytische Differenzierung von „Manipulation“ und „Konstruktion“ von Wissen und Wirklichkeit - als eine genuin wissenssoziologischkonstruktivistische Problematik- eine oft sehr heikle ist.

\section{References}

1. Douglas, R. M.; Hemilä, H. (2005): Vitamin C for Preventing and Treating the Common Cold. PLoS Med, 2 (6). Verfügbar unter: https://doi.org/10.1371/journal.pmed.0020168.

2. Zahlen nach dem Lebensmittelverband Deutschland (2015): Studie: Bewusster Umgang mit Nahrungsergänzungsmitteln. Repräsentative Umfrage zur Anwendungshäufigkeit. Verfügbar unter: https://www.lebensmittelverband.de/de/aktuell/20151110-studie-bewussterumgang-verwendung-nahrungsergaenzungsmittel.

3. Vgl. Meier, E. (1954): Alfred Nobel: Nobelstiftung, Nobelpreise. Berlin: Duncker \& Humblot.

4. Zit. n. Bächi, B. (2009): Vitamin C für alle! Pharmazeutische Produktion, Vermarktung und Gesundheitspolitik (1933-1953). Zürich: Chronos. S. 9. Verfügbar unter: https://doi.org/10.3929/ethz-a-005788850.

5. Sämtliche Zitate nach: A.a.O.: S. 130-141.

6. A.a.O.: S. $142 \mathrm{f}$.

7. Thomas, W.I.; Thomas, D.S. (1928): The child in America: Behavior problems and programs. New York: Knopf, S. $571 \mathrm{f}$.

8. Berger, P.; Luckmann, T. (1980): Die gesellschaftliche Konstruktion der Wirklichkeit. Frankfurt a.M.: S. Fischer. S. 3.

9. Wenn nämlich, wie Helmut Plessner in dem Kommentar zur deutschen Ausgabe von Berger und Luckmann schreibt, das „Element der Konstruktion [...] als Widersacher jedes wissenschaftlichen Bemühens empfunden [wird]“ (a.a.O.: S. XI).

SUGGESTED CITATION: Hartwig, Sebastian Till: Konstruktionen medizinischer Wirklichkeit, in: KWI-BLOG, [https://blog.kulturwissenschaften.de/konstruktionenmedizinischer-wirklichkeit/l, 22.06.2020

DOI: https://doi.org/10.37189/kwi-blog/20200622-0900 


\section{DuEPublico}

offen im Denken

Duisburg-Essen Publications online Ub | universitäts

Dieser Text wird über DuEPublico, dem Dokumenten- und Publikationsserver der Universität Duisburg-Essen, zur Verfügung gestellt. Die hier veröffentlichte Version der EPublikation kann von einer eventuell ebenfalls veröffentlichten Verlagsversion abweichen.

DOI: $\quad$ 10.37189/kwi-blog/20200622-0900

URN: urn:nbn:de:hbz:464-20200622-112058-7

Alle Rechte vorbehalten. 